\title{
Flight Evaluation of an LPV Sliding Mode Controller with Online Control Allocation
}

\author{
L. Chen*, H. Alwi*, C. Edwards* and M. Sato**
}

\begin{abstract}
This paper presents the results of flight tests of a fault tolerant sliding mode controller implemented on the Japan Aerospace Exploration Agency's Multi-Purpose Aviation Laboratory aircraft. These represent the first validation tests of a sliding mode control allocation scheme on a piloted flight test. In this scheme, information about the actuator faults is assumed to be estimated online from a fault detection unit and the available actuators are fully utilized in the presence of actuator faults, in an effort to retain nominal fault free performance. Specifically the flight tests results demonstrate good lateral-directional state tracking performance in the fault free case with no visible performance degradation in the presence of rudder and aileron faults. In fact, during the flight test, the evaluation pilot did not detect any degradation in manoeuvrability when the actuator faults occurred.
\end{abstract}

\section{INTRODUCTION}

In the last decade, fault detection and diagnosis (FDD), and fault tolerant control (FTC) schemes have been widely developed within the academic community. However, most of the results were only demonstrated in simulation and there remains a significant theory/practice gap. Some recent EU funded projects allowed FDD/FTC approaches to be verified and validated at a system integration level. For example, FTC approaches were validated on the SIMONA motion simulator during the GARTEUR FM-AG16 project [1]; FDD approaches were validated and verified using the Airbus actuator integration bench and 'Ironbird' platform at Toulouse during ADDSAFE [2], and the FTC/FDD approaches were validated and demonstrated using the Airbus desktop simulator during the RECONFIGURE project [3]. Although the gap between the advanced FDD/FTC approaches developed from academic community and industry demands have been bridged due to these system integration level implementations, very few FDD/FTC schemes have been validated in actual flight test, which is usually considered as the top level task of the system integration. A notable exception is the bespoke propulsion controlled aircraft (PCA) system developed by NASA Dryden in the 1990's which was implemented and flight tested on a MD-11 and a F-15 aircraft [4], [5].

Any approaches being integrated during flight tests must have the capabilities to deal with various system uncertainties, such as external disturbances induced by wind or gusts, actuator delays and modelling uncertainties. Thanks to the recent H2020/Japan co-funded project VISION (Validation of Integrated Safety-enhanced Intelligent flight cONtrol) project, sliding mode based FDD/FTC approaches, developed by the University of Exeter, were validated during flight tests on the Japan Aerospace Exploration Agency's Multi-purpose Aviation Laboratory (MuPAL- $\alpha$ ) aircraft [6], [7]. Some of these recent results will be presented for the first time in this paper.

Sliding mode based control schemes have an inherent capability to reject so-called matched uncertainty i.e. uncertainty which occurs in the channels in which the control signals act. It is easy to recognize that actuator faults and failures, by their very definition, act in these channels and therefore can be considered to be a very particular form of matched uncertainty. From this observation, sliding mode controllers are a natural candidate for use as fault tolerant controllers. Recent work has demonstrated the theoretical benefits of adroitly employing sliding mode controllers within a control allocation framework for overactuated systems [8], [9]. In this paper, online control allocation is exploited as part of the sliding mode

\footnotetext{
* College of Engineering, Mathematics \& Physical Sciences, University of Exeter, UK. lc427@exeter.ac.uk, h.alwidexeter.ac.uk, C.Edwards@exeter.ac.uk

** Japan Aerospace Exploration Agency, Mitaka, Tokyo 181-0015, Japan. sato.masayuki a jaxa.jp
} 
control scheme and the actuator effectiveness levels are explicitly used as part of control laws in order to fully utilize the availability of the actuators.

In recent years, linear parameter varying (LPV) based synthesis methodologies [10], [11], [12], [18] have been widely considered in aerospace FDD/FTC applications. This is because the LPV framework allows robustness and stability to be ensured over a wide range of flight conditions. In this paper, a new LPV sliding mode FTC scheme is developed and implemented within MuPAL- $\alpha$ 's experimental Fly-By-Wire (FBW) system. The proposed scheme combines the inherent robustness of sliding mode control (SMC) against uncertainty and actuator faults/failures, and an online control allocation (CA) structure that fully utilizes the available redundancy within the system and preferentially redistributes the control signals to healthy actuators, based upon knowledge from the FDI component. This allocation scheme allows the FTC performance to be improved due to reduced design conservatism, whilst also ensuring stability and robustness over a wide range of the flight envelope. This paper focusses on lateral-directional control and fault scenarios wherein the ailerons and the rudder are assumed to work at a reduced effectiveness levels. Although the scheme does have the potential to cope with total rudder failure, this has not been pursued in this particular publication.

The main contribution of the paper is that for the first time a sliding mode control allocation scheme has been implemented and validated during a piloted flight test on a full scale aircraft. Furthermore, the FTC scheme is developed in an LPV framework, which allows the nominal fault free performance to be retained in the presence of the faults/failures over a wide range of flight conditions. The proposed scheme is different from the existing work in [13], [14] because the sliding surface is also dependent on the scheduling parameter.

\section{LPV SLIDING MODE CONTROLLER DESIGN}

\section{A. Problem formulation}

In this section, an LPV sliding mode FTC scheme with online CA is developed from a theoretical standpoint. Consider an LPV system with actuator faults/failures given by

$$
\begin{aligned}
\dot{x}_{p}(t) & =A_{p}(\rho) x_{p}(t)+B_{p}(\rho)\left(I_{m}-K(t)\right) u_{p}(t)+D_{p}(\rho) \xi(t) \\
y_{c}(t) & =C_{c} x_{p}(t)
\end{aligned}
$$

where $A(\rho) \in \mathbb{R}^{n \times n}, B(\rho) \in \mathbb{R}^{n \times m}, C_{c} \in \mathbb{R}^{l \times n}$ and $l<m$. The state vector and the control input are denoted by $x_{p} \in \mathbb{R}^{n}$ and $u_{p} \in \mathbb{R}^{m}$ respectively. The disturbance distribution matrix $D_{p}(\rho) \in \mathbb{R}^{n \times k}$ and $\xi(t) \in \mathbb{R}^{k}$ denotes the 'matched' disturbance. The time varying scheduling parameter $\rho \in \mathbb{R}^{n_{r}}$ is assumed to be available and lie in a hyper-rectangle $\Omega \subset \mathbb{R}^{n_{r}}$. In (1), the weighting matrix $K(t):=$ $\operatorname{diag}\left(k_{1}(t), \ldots, k_{m}(t)\right)$ where the time varying scalars $k_{1}, k_{2}, \ldots k_{m}$ represent the loss of effectiveness of the actuators [13]. For a fault-free actuator $k_{j}=0$ and for a completely failed actuator $k_{j}=1$. When $0<k_{j}<1$, the actuator behaves with reduced effectiveness (i.e. a fault). Since it is assumed there are only $l<m$ controlled outputs, there is redundancy in the system. This will be exploited for fault tolerant control.

To introduce tracking, define integrator states as

$$
\dot{x}_{r}(t)=r(t)-C_{c} x_{p}(t)
$$

where $r(t)$ is the (differentiable) command signal which satisfies the pre-filter equations

$$
\dot{r}(t)=\Gamma\left(r(t)-R_{c}\right)
$$

In (3), $R_{c}$ represents a fixed demand vector and $\Gamma \in \mathbb{R}^{l \times l}$ is a stable design matrix.

Let $W(t) \in \mathbb{R}^{m \times m}$ be defined by

$$
W(t):=I_{m}-K(t)
$$

where the $i$ th diagonal element is $w_{i}(t)=1-k_{i}(t)$. 
Let $x_{a}(t)=\operatorname{col}\left(x_{r}(t), x_{p}(t)\right)$ and consider the augmented state space system

$$
\dot{x}_{a}(t)=\underbrace{\left[\begin{array}{cc}
0 & -C_{c} \\
0 & A_{p}(\rho)
\end{array}\right]}_{A_{a}(\rho)} x_{a}(t)+\underbrace{\left[\begin{array}{c}
0 \\
B_{p}(\rho)
\end{array}\right]}_{B_{a}(\rho)} W(t) u(t)+\underbrace{\left[\begin{array}{c}
I_{l} \\
0
\end{array}\right]}_{B_{c}} r(t)+\underbrace{\left[\begin{array}{c}
0 \\
D_{p}(\rho)
\end{array}\right]}_{D_{a}(\rho)} \xi(t)
$$

Assumption 2.1: Assume $B_{a}(\rho)$ can be factorized as

$$
B_{a}(\rho)=B_{v} B_{2}(\rho)
$$

where $B_{v} \in \mathbb{R}^{(n+l) \times l}$ is a fixed matrix, and $B_{2}(\rho) \in \mathbb{R}^{l \times m}$ is a matrix with varying components where $\operatorname{rank}\left(B_{2}(\rho)\right)=l$ for all $\rho \in \Omega$ and $\operatorname{rank}\left(B_{v}\right)=l$.

This factorization assumption is common in the LTI system literature when considering control allocation and systems with redundancy - especially aircraft (see for example [15], [16]). The factorization will allow a 'virtual' control to be designed which is of lower order than the total number of the redundant actuators in the system and control allocation will provide a means of distributing the control efforts. This factorization is discussed (in the LTI case) in [15] together with the implicit assumption on rank which this imposes on the matrix $B_{a}$.

Since $\operatorname{rank}\left(B_{v}\right)=l$ there exists a coordinate change $x_{a} \mapsto T_{a} x_{a}$ where

$$
T_{a}=\left[\begin{array}{cc}
I_{l} & 0 \\
0 & T_{n}
\end{array}\right]
$$

and $T_{n} \in \mathbb{R}^{n \times n}$ such that

$$
T_{n} B_{v}=\left[\begin{array}{l}
0 \\
I_{l}
\end{array}\right]
$$

In the sliding mode literature this separation of the input distribution matrix creates so-called regular form [17]. Then (5) can be written as

$$
\dot{x}(t)=A(\rho) x(t)+\left[\begin{array}{c}
0 \\
B_{2}(\rho)
\end{array}\right] W(t) u(t)+B_{c} r(t)+D(\rho) \xi(t)
$$

where $A(\rho)=T_{a} A_{a}(\rho) T_{a}^{-1}$ and $D(\rho)=T_{a} D_{a}(\rho)$.

Define a virtual control signal $v(t) \in \mathbb{R}^{l}$ (nominally) as

$$
v(t):=B_{2}(\rho) u(t)
$$

If the actual physical control signals sent to the actuators are given by

$$
u(t):=B_{2}(\rho)^{\dagger} v(t)
$$

where

$$
B_{2}(\rho)^{\dagger}:=\Lambda(t) B_{2}(\rho)^{T}\left(B_{2}(\rho) \Lambda(t)^{2} B_{2}(\rho)^{T}\right)^{-1}
$$

and $\Lambda(t)$ is a weighting matrix, then when $\Lambda(t)=I, B_{2}(\rho)^{\dagger}$ is a pseudo-inverse of $B_{2}(\rho)$. In this paper, for the purpose of developing an online control allocation scheme, it is assumed $\Lambda(t)$ is an online estimate of $W(t)$. Specially assumed

$$
\Lambda(t):=(I+\Delta(t))^{-1} W(t)
$$

where the diagonal matrix $\Delta(t) \in \mathbb{R}^{m \times m}$ encapsulates the uncertainty in the estimate of $W(t)$ by $\Lambda(t)$. Define the admissible set $\mathcal{W}$ as

$$
\mathcal{W}=\left\{W(t) \subseteq \operatorname{diag}\left(w_{1}, \ldots, w_{m}\right), \quad 0 \leq w_{i} \leq 1\right\}
$$

such that for all $W(t) \in \mathcal{W}$ and $\rho \in \Omega$,

$$
\operatorname{det}\left(B_{2}(\rho) W(t) B_{2}(\rho)^{T}\right) \neq 0
$$


Assumption 2.2: Assume the uncertainty $\Delta(t)$ satisfies

$$
\|\Delta(t)\|<\frac{1-\Delta_{\max }}{\left\|B_{2}(\rho)\right\|\left\|B_{2}(\rho)^{\dagger}\right\|}
$$

for all $W(t) \in \mathcal{W}$ and $\rho \in \Omega$ where $0<\Delta_{\max }<1$.

From (13) if $\Delta(t)=0, \Lambda(t)=W(t)$ and then substituting (11) and (12) into (9) yields

$$
\dot{x}(t)=A(\rho) x(t)+\left[\begin{array}{l}
0 \\
I_{l}
\end{array}\right] v(t)+B_{c} r(t)+D(\rho) \xi(t)
$$

The design problem is to create a virtual control law $v(t)$ to ensure closed-loop stability of the system in (17) for all combinations of faults/failures belonging to $\mathcal{W}$.

Partition the states in (17) as $x(t)=\operatorname{col}\left(x_{1}(t), x_{2}(t)\right)$ where $x_{1}(t) \in \mathbb{R}^{n}$ and $x_{2}(t) \in \mathbb{R}^{l}$ then (17) in partitioned form becomes

$$
\left[\begin{array}{l}
\dot{x}_{1}(t) \\
\dot{x}_{2}(t)
\end{array}\right]=\left[\begin{array}{ll}
A_{11}(\rho) & A_{12}(\rho) \\
A_{21}(\rho) & A_{22}(\rho)
\end{array}\right]\left[\begin{array}{l}
x_{1}(t) \\
x_{2}(t)
\end{array}\right]+\left[\begin{array}{l}
0 \\
I_{l}
\end{array}\right] v(t)+\left[\begin{array}{l}
B_{c 1} \\
B_{c 2}
\end{array}\right] r(t)+\left[\begin{array}{l}
D_{1}(\rho) \\
D_{2}(\rho)
\end{array}\right] \xi(t)
$$

Since $\xi(t)$ is 'matched' uncertainty, in (18) $D_{1}(\rho)=0$.

Assumption 2.3: Assume $\left(A(\rho), B_{v}\right)$ is controllable (or equivalently $\left(A_{11}(\rho), A_{12}(\rho)\right)$ is controllable).

\section{B. Definition of the switching function}

Define a parameter-dependent switching function as

$$
s(t)=S(\rho) x(t)
$$

where

$$
S(\rho)=\left[M(\rho) \quad I_{l}\right]
$$

and $M(\rho) \in \mathbb{R}^{l \times n}$ represents the design degree of freedom. Assume

$$
\begin{aligned}
A(\rho) & =A_{0}+A_{1} \rho_{1}(t) \ldots+A_{n_{r}} \rho_{n_{r}}(t) \\
M(\rho) & =M_{0}+M_{1} \rho_{1}(t) \ldots+M_{n_{r}} \rho_{n_{r}}(t)
\end{aligned}
$$

During sliding $s(t)=0$ and therefore from (20)

$$
x_{2}(t)=-M(\rho) x_{1}(t)
$$

Substituting (22) into (18) yields the reduce order sliding motion

$$
\dot{x}_{1}(t)=\hat{A}_{11}(\rho) x_{1}(t)+B_{c 1} r(t)
$$

where $\hat{A}_{11}(\rho)=A_{11}(\rho)-A_{12}(\rho) M(\rho)$. Since $\hat{A}_{11}(\rho)$ is dependent on $M(\rho)$, the choice of $M(\rho)$ may be viewed as a parameter-dependent state feedback problem for the pair $\left(A_{11}(\rho), A_{12}(\rho)\right)$. Since the scheduling parameter $\rho$ varies inside a polytope and $(A(\rho), M(\rho))$ affinely depend on $\rho$ (as shown in (21)), then the parameter-dependent state feedback problem can be solved using the vertex property [18]. Define $\Psi_{i j}=A_{11, i} P_{1}-A_{12, i} Q_{j}$ where $A_{11, i}$ and $A_{12, i}$ in $\Psi_{i j}$ represent the $i$ th and $j$ th vertex of $A_{11}(\rho)$ and $A_{12}(\rho)$, respectively, and in general

$$
\left[\begin{array}{c}
A_{11}(\rho) \\
A_{12}(\rho) \\
M(\rho)
\end{array}\right]=\sum_{i=1}^{N} p_{i}(\rho)\left[\begin{array}{c}
A_{11, i} \\
A_{12, i} \\
\bar{M}_{i}
\end{array}\right]
$$

with

$$
\sum_{i=1}^{N} p_{i}(\rho)=1 \quad \text { and } \quad p_{i}(\rho) \geq 0
$$


If there exist a symmetric positive definite matrix $P_{1}$ and $Q_{j}$ such that the LMIs (26) and (27) are feasible,

$$
\begin{gathered}
{\left[\begin{array}{cc}
-P_{1} & \Psi_{i j} \\
\Psi_{i j}^{T} & -P_{1}
\end{array}\right]<0 \quad \forall i, j=1, \ldots, N} \\
\alpha \otimes P_{1}+\beta \otimes \Psi_{i j}+\beta^{T} \otimes \Psi_{i j}^{T}<0 \forall i, j=1, \ldots, N
\end{gathered}
$$

where $N=2^{n_{r}}$, then equation (27) indicates that $\Psi_{i j}$ is $\mathcal{D}$-stable and the region $\mathcal{D}$ is specified by a pair of $\alpha$ and $\beta$. See [18] for more details. Then the $j$ th vertex of $M(\rho)$ can be calculated as $\bar{M}_{j}=Q_{j} P_{1}^{-1}$ for $j=1, \ldots, N$ and $M(\rho)$ is given by

$$
M(\rho)=\sum_{j=1}^{N} p_{j}(\rho) \bar{M}_{j}
$$

\section{Control law with online control allocation}

In this section, a control law is selected to ensure and maintain the sliding motion. Here the virtual control law contains two components: a 'linear' component $v_{l}(t)$ and a 'nonlinear' component $v_{n}(t)$. Specifically:

$$
v(t)=v_{l}(t)+v_{n}(t)
$$

where

$$
v_{l}(t)=-S(\rho)\left(A(\rho) x(t)+B_{c} r(t)\right)-\left(M(\dot{\rho})-M_{0}\right) x_{1}(t)+\Phi s(t)
$$

and $\Phi \in \mathbb{R}^{l \times l}$ is a Hurwitz matrix whilst the term

$$
v_{n}(t)=-\mathcal{K}(t, x) \frac{P_{2} s(t)}{\left\|P_{2} s(t)\right\|} \quad \text { if } \quad s(t) \neq 0
$$

where the symmetric positive definite matrix $P_{2}$ satisfies

$$
P_{2} \Phi+\Phi^{T} P_{2}=-I_{l}
$$

Assumption 2.4: Assume the disturbance signal $\xi(t)$ in (1) is bounded and satisfies

$$
\|\xi(t)\| \leq \alpha(t, x)
$$

Suppose the modulation gain in (31) is chosen as

$$
\mathcal{K}(t, x) \geq \frac{\left\|D_{2}(\rho)\right\| \alpha(t, x)+\eta+\left(1-\Delta_{\max }\right)\left\|v_{l}\right\|}{\Delta_{\max }}
$$

where $\eta$ is a positive scalar. It will now be shown that the control law $v(t)$ in (29)-(31) induces and maintains a sliding motion on $s(t)=0$ in finite time.

From (19) and (20), in the general case when $\Delta(t) \neq 0$, using (9)-(13), the derivative of $s(t)$ is given by

$$
\begin{aligned}
\dot{s}(t) & =S(\rho) \dot{x}(t)+\frac{\partial S(\rho)}{\partial \rho} \dot{\rho} x(t) \\
& =S(\rho) \dot{x}(t)+\left(M(\dot{\rho})-M_{0}\right) x_{1}(t) \\
& =S(\rho)\left(A(\rho) x(t)+B_{c} r(t)\right)+v(t)+B_{2}(\rho) \Delta B_{2}(\rho)^{\dagger} v(t)+D_{2}(\rho) \xi(t)+\left(M(\dot{\rho})-M_{0}\right) x_{1}(t)
\end{aligned}
$$

Substituting (30) and (31) into (35) yields the reduced order dynamics

$$
\dot{s}(t)=\Phi s(t)-\mathcal{K}(t, x) \frac{P_{2} s(t)}{\left\|P_{2} s(t)\right\|}+D_{2}(\rho) \xi(t)+B_{2}(\rho) \Delta(t) B_{2}(\rho)^{\dagger} v(t)
$$

Now consider a quadratic Lyapunov function given by

$$
V(s)=s^{T} P_{2} s
$$


Differentiating $V(s)$ yields

$$
\dot{V}(s)=s^{T}\left(\Phi^{T} P_{2}+P_{2} \Phi\right) s-2 \mathcal{K}\left\|P_{2} s\right\|+2 s^{T} P_{2} D_{2}(\rho) \xi(t)+2 s^{T} P_{2} B_{2}(\rho) \Delta(t) B_{2}(\rho)^{\dagger} v(t)
$$

Using the fact that $P_{2} \Phi+\Phi^{T} P_{2}=-I_{l}$, it follows

$$
\dot{V}(s)=-\|s\|^{2}-2 \mathcal{K}\left\|P_{2} s\right\|+2 s^{T} P_{2} D_{2}(\rho) \xi(t)+2 s^{T} P_{2} B_{2}(\rho) \Delta(t) B_{2}(\rho)^{\dagger} v(t)
$$

It follows from Assumption 2.2 that

$$
\left\|B_{2}(\rho) \Delta(t) B_{2}(\rho)^{\dagger}\right\|<\left\|B_{2}(\rho)\right\|\|\Delta(t)\|\left\|B_{2}(\rho)^{\dagger}\right\|<1-\Delta_{\max }
$$

and therefore if $d_{2}=\max _{\rho \in \Omega}\left\|D_{2}(\rho)\right\|$

$$
\begin{aligned}
\dot{V} & \leq-\|s\|^{2}-2\left\|P_{2} s\right\|\left(\mathcal{K}-d_{2}\|\xi\|-\left(1-\Delta_{\max }\right)\|v\|\right) \\
& \leq-\|s\|^{2}-2\left\|P_{2} s\right\|\left(\mathcal{K}-d_{2}\|\xi\|-\left(1-\Delta_{\max }\right)\left(\left\|v_{l}\right\|+\left\|v_{n}\right\|\right)\right) \\
& \leq-\|s\|^{2}-2\left\|P_{2} s\right\|\left(\mathcal{K}-d_{2}\|\xi\|-\left(1-\Delta_{\max }\right)\left(\left\|v_{l}\right\|+\mathcal{K}\right)\right) \\
& \leq-\|s\|^{2}-2\left\|P_{2} s\right\|\left(\Delta_{\max } \mathcal{K}-d_{2}\|\xi\|-\left(1-\Delta_{\max }\right)\left\|v_{l}\right\|\right)
\end{aligned}
$$

Substituting (34) into (41) yields

$$
\dot{V}(s) \leq-\|s\|^{2}-2 \eta\left\|P_{2} s\right\|
$$

and using the Rayleigh principle

$$
\dot{V}(s) \leq-2 \bar{\eta} \sqrt{V}
$$

where

$$
\bar{\eta}=\eta \sqrt{\lambda_{\min }\left(P_{2}\right)}
$$

Since $\bar{\eta}>0$, inequality (43) implies $V=0$ in finite time and therefore $s=0$ in finite time and sliding is attained and can maintained for all subsequent time.

Finally, the actual input $u_{p}$ using online control allocation is given by

$$
u_{p}=\Lambda(t) B_{2}(\rho)^{T}\left(B_{2}(\rho) \Lambda(t)^{2} B_{2}(\rho)^{T}\right)^{-1} v(t)
$$

where $v(t)$ is defined in (29).

\section{Flight test ON MuPAL- $\alpha$}

\section{A. $M U P A L-\alpha$}

The JAXA Multi-Purpose Aviation Laboratory (MuPAL- $\alpha$ ) research aircraft (Fig. 1) is based on the 2propeller engine Dornier Do228-202 aircraft. It has been modified to include a research fly-by-wire (FBW) system and Direct Lift Control (DLC) (Fig. 1(b)). MuPAL- $\alpha$ has been extensively used for evaluating human-machine interactions, and testing state-of-the-art control, guidance and navigation technologies - including fault tolerant control strategies [7], [20]. The FBW configuration allows new flight control strategies to be implemented (on the FBW flight control computer) and then evaluated either on the ground (as part of a hardware-in-the-loop configuration) or by actual flight evaluation.

The flight crew (see Fig. 1(b)) includes the evaluation and safety pilots (on the right and left side of the cockpit respectively) as well as FBW, data acquisition and flight guidance operators. 


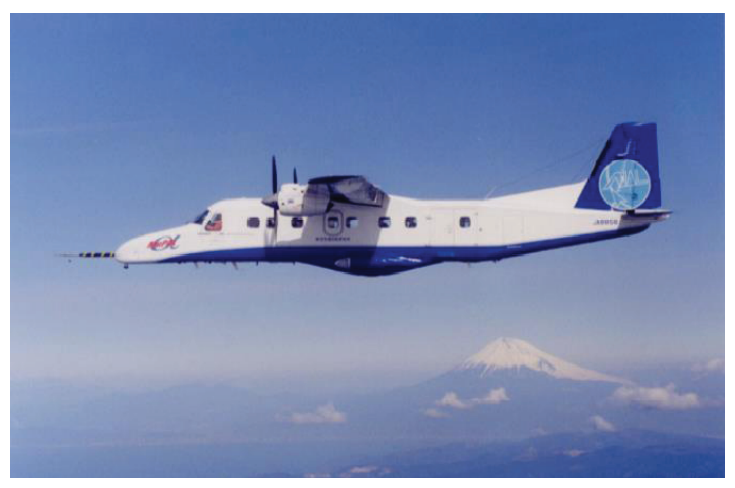

(a) aircraft

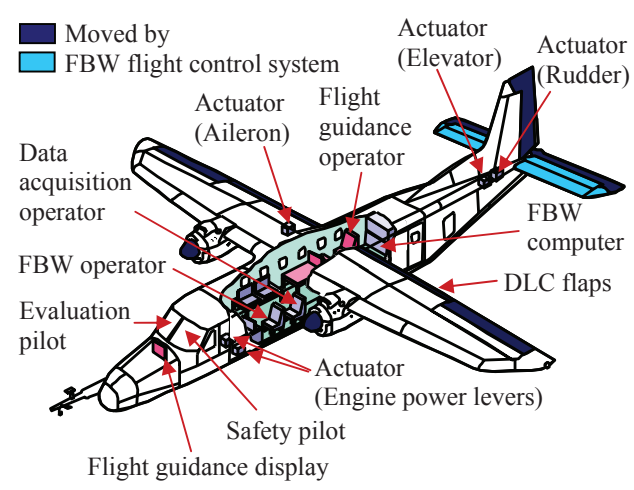

(b) fly-by-wire configuration

Fig. 1. MuPAL- $\alpha$ aircraft

\section{B. Controller Design}

The LPV model of MuPAL- $\alpha$ was created via interpolation of a collection of linear time invariant (LTI) models provided by JAXA. The model is based around an altitude $5000 \mathrm{ft}$ with standard atmospheric conditions. The LPV model is scheduled against the indicated airspeed according to

$$
\rho=\left[\begin{array}{ll}
\rho_{1} & \rho_{2}
\end{array}\right]=\left[\begin{array}{ll}
v_{\text {ias }} & v_{\text {ias }}^{2}
\end{array}\right]
$$

where $v_{\text {ias }}$ represents the indicated airspeed. Here, for design purposes, a conservative assumption were made where the scheduling parameters $\rho_{1}$ and $\rho_{2}$ are assumed to be independent of each other (although in this case, they are actually related through $v_{\text {ias }}$ ). For controller synthesis purposes, both scheduling parameters have been scaled to the range $\left[\begin{array}{ll}0 & 1\end{array}\right]$.

This paper will focus on lateral-directional control. The system states are roll angle $\phi$, sideslip angle $\beta$, yaw rate $r$ and roll rate $p$, respectively. The system inputs are differential trust $\delta_{t d}$, the aileron $\delta_{a}$ and rudder surface deflections $\delta_{r}$. The command signals are the sideslip angle $\beta_{c}$ and the roll angle commands $\phi_{c}$ respectively.

Remark 3.1: Note that during the flight test, differential trust is not used for control allocation - although in future subsequent flight trials will investigate this possibility.

As in other typical aircraft, the force derivatives in the $Y$ component associated with the control surfaces (given by the term $\frac{Y_{\delta_{a}}}{u_{0}}$ and $\frac{Y_{\delta_{r}}}{u_{0}}$ and associated with the state $\beta$ ) are much smaller than the moments control derivatives components $M$ and $N$ (associated with the state $p$ and $r$ respectively). Therefore, as in [16], [21], for the purpose of controller synthesis, the term $\frac{Y_{\delta_{a}}}{u_{0}}$ and $\frac{Y_{\delta_{r}}}{u_{0}}$ are neglected. This allows the perfect factorization of $B(\rho)$ as

$$
B(\rho)=B_{v} B_{2}(\rho)
$$


where

$$
B_{v}=\left[\frac{0_{4 \times 2}}{I_{2}}\right] \quad \text { and } \quad B_{2}(\rho)=\left[\begin{array}{lll}
b_{51}(\rho) & b_{52}(\rho) & b_{53}(\rho) \\
b_{61}(\rho) & b_{62}(\rho) & b_{63}(\rho)
\end{array}\right]
$$

and $B_{2}(\rho) \in \mathbb{R}^{2 \times 3}$. This structure is amenable to the use of the controller described in the earlier sections. To ensure a stable reduced order sliding motion in (23), the $\mathcal{D}$-stable region is selected to the left of the vertical line through -1 in the complex plane. In this case, $\alpha$ and $\beta$, defined in (27), are chosen as $\alpha=-2$ and $\beta=1$. All the flight tests have been conducted at a fixed height with an initial indicated airspeed around 120kts. Since during this flight test, the airspeed is slow-varying in a limited range, the value of $M(\rho)$ has been fixed at

$$
M(\rho)=\left[\begin{array}{cccc}
2.8459 & -0.4797 & 0.3176 & -2.7233 \\
-0.5889 & -2.7035 & 2.8174 & 0.4558
\end{array}\right]
$$

For implementation purposes, a 'smooth' approximation of the discontinuous output injection signal from (31) is used [22], specifically

$$
v_{n}=-\mathcal{K}(t, x) \frac{P_{2} s(\rho)}{\left\|P_{2} s(\rho)\right\|+\delta}
$$

where the modulation gain $\mathcal{K}=0.4$ and the smoothing factor $\delta=0.05$. In (32), the Lyapunov matrix $P_{2}$ and $\Phi$ are selected as

$$
P_{2}=I_{2} \quad \text { and } \Phi=-0.5 I_{2}
$$

\section{Controller implementation}

The proposed control scheme was coded in $\mathrm{C}$ and implemented on board the MuPAL- $\alpha$ flight control computer. The controller was first ground tested (in a hardware-in-the-loop configuration) for pre-flight checks and to allow the crew to become familiar with the scheme in preparation for the actual flight tests. In this first flight tests campaign, the faults were implemented at a software level, rather than at a hardware level, for safety reasons. The output signal from the controller is passed through a gain (at a software level) to simulate a reduction in actuator effectiveness (i.e. fault), and it is this signal which is sent to the actuators (rudder and ailerons).

No FDI scheme has been incorporated yet within the flight control computer. In all the flight tests an estimate of the fault

$$
\widehat{W}=\widehat{G}(s) W
$$

where $\widehat{G}(s)$ represents a low pass lag filter to simulate the presence of a fault estimation scheme and thus when a fault is initially introduced $\widehat{W}(t) \neq W(t)$ but $\widehat{W}(t) \rightarrow W(t)$ as $t \rightarrow \infty$. In all the flight tests $\Lambda(t)=\widehat{W}$ Note that during the flight test, it is assumed faults are known, i.e. $\Delta=W$. The FDI scheme will be included in the future flight tests.

\section{Flight tests campaign}

The results presented in the following subsections were obtained from flight test campaigns manned by a crew of JAXA and Nikanihon Air Service personnel, between 16-27 January 2017 to evaluate the FTC scheme. The MuPAL- $\alpha$ took off from Chofu airport (see Fig. 2) near JAXA's Mitaka base. The flight tests took place in Sagami Bay, south-west of Tokyo (see Fig. 2). The weather conditions were clear and satisfied the visual meteorological conditions (VMC) flight category (which allowed the flight test to take place). During the test, the maximum recorded wind/gust was $11.3652 \mathrm{~m} / \mathrm{s}$.

Since this flight test campaign concentrated on the evaluation of the lateral-directional control, the longitudinal control of altitude and speed is manually maintained by the evaluation pilot through the column and throttle lever inputs. For the evaluation of the proposed lateral-directional controller, the commands are created manually by the evaluation pilot. For comparison purposes, a coordinated 'S-turn' manoeuvre (see Fig. 3 with a roll angle of $\pm 20 \mathrm{deg}$ was conducted during the flight test campaign. Figure 3 represents snapshots from a video recorded during one of the flight tests. 


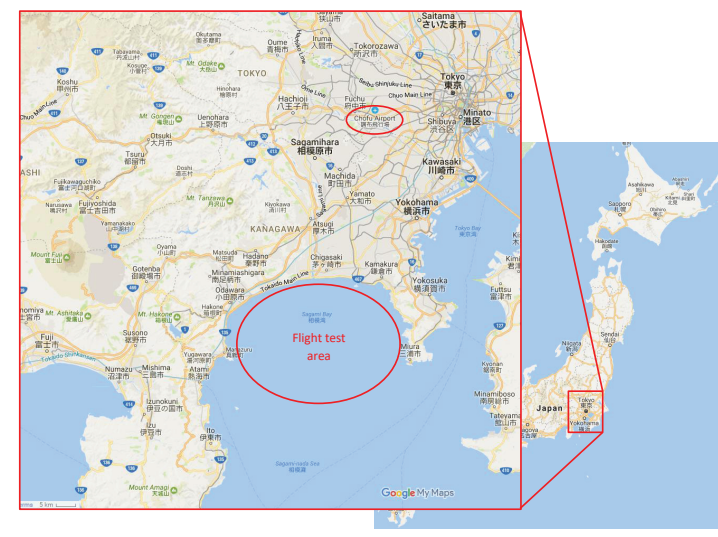

Fig. 2. Chofu airport and Sagami Bay (flight test area)

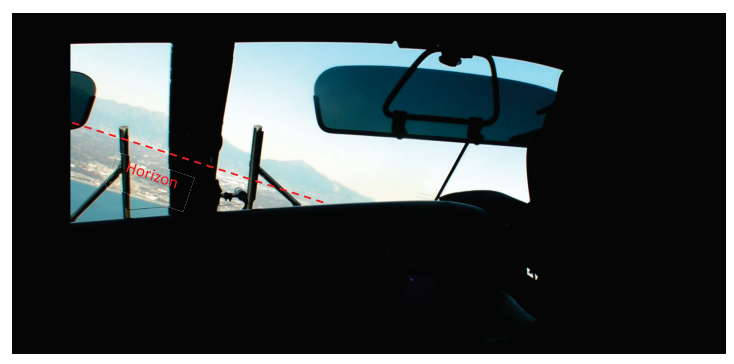

Fig. 3. Video snapshot from inside the cockpit during the flight test (coordinated 'S-turn')

1) Simultaneous aileron and rudder faults: Figure 4 illustrates the flight test results when faults occur on the aileron and the rudder simultaneously. Here the aileron and the rudder work at $70 \%$ efficiency and $90 \%$ efficiency respectively. In this case, $K=\operatorname{diag}(1,0.3,0.1)$. The trajectories of the lateral-directional states are also shown in Fig. 4 wherein a steady turn manoeuvre with $\pm 20 \mathrm{deg}$ roll angle commands is created by the evaluation pilot. Clearly, although there exist simultaneous rudder and aileron faults, the proposed scheme can still achieve good roll and sideslip tracking performance. Furthermore, Figure 4 also shows that, during the flight test, sliding is maintained. The aileron and rudder commands and their surface deflections are also shown in Fig. 4. It can be seen that the aileron and the rudder cannot follow the respective demands due to the existence of faults.

\section{CONCLUSION}

This paper has developed an LPV sliding mode control allocation scheme in which online control allocation is used to fully utilize the actuator availability in the face of actuator faults, based on knowledge from the FDI component. The proposed scheme has been implemented on the Japan Aerospace Exploration Agency Multi-purpose Aviation Laboratory (MuPAL- $\alpha$ ) research aircraft and validated during flight tests. The illustrated flight test results show that, during the steady turn manoeuvre induced by the evaluation pilot, roll and sideslip tracking performance is maintained in the face of simultaneous rudder and aileron faults.

\section{ACKNOWLEDGEMENTS}

This research has received funding from the H2020 innovation programme under grant agreement No. 690 811, and from the Japan New Energy and Industrial Technology Development Organization under grant agreement No. 062800, as the research project entitled 'Validation of Integrated Safety-enhanced Intelligent flight cONtrol (VISION)'. 

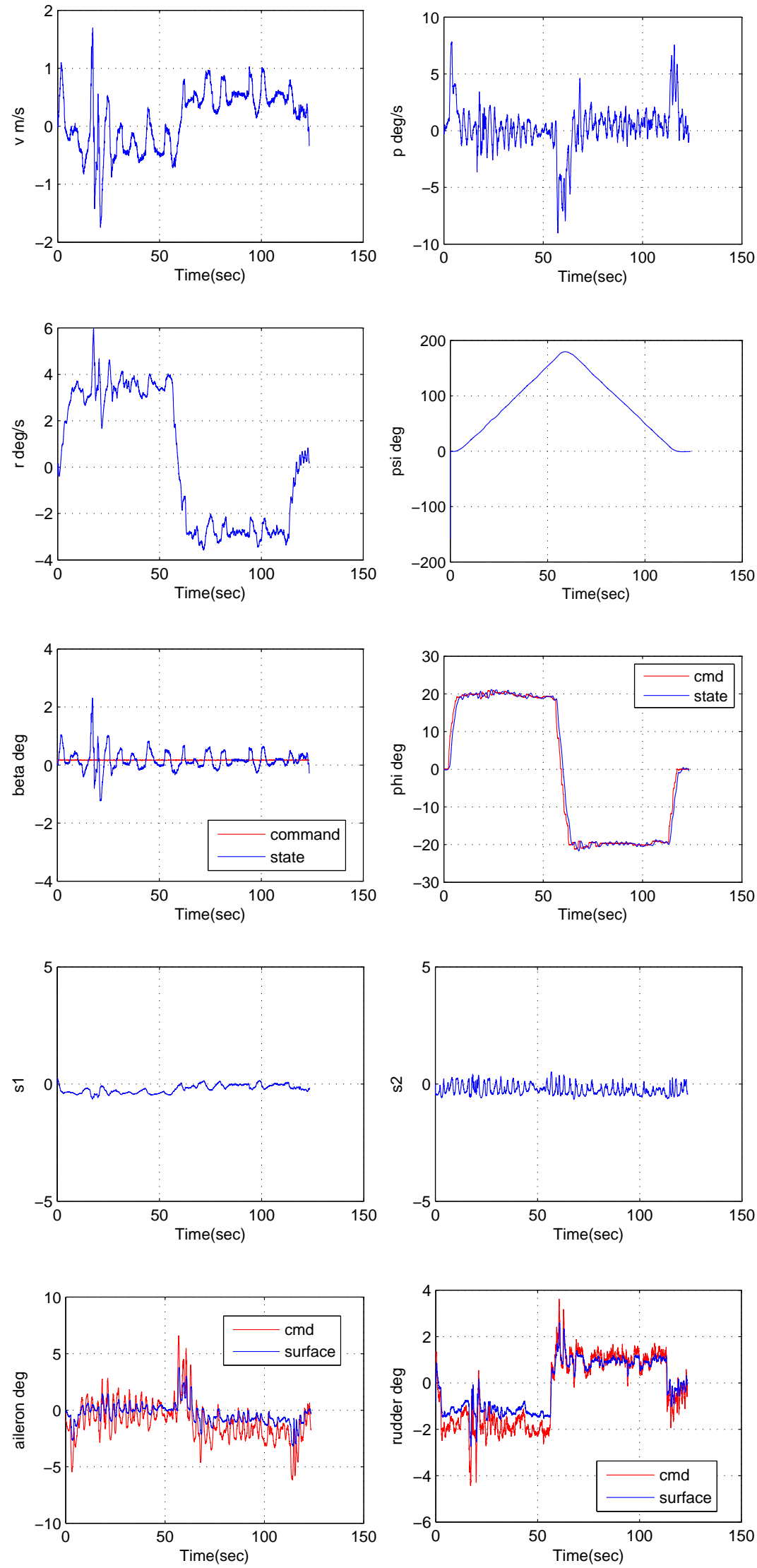

Fig. 4. Aileron and rudder faults $-K=\operatorname{diag}(1,0.3,0.1)$ : states, switching functions and control surface deflections 


\section{REFERENCES}

[1] C. Edwards, T. Lombaerts, and H. Smaili, Fault tolerant flight control: A benchmark challenge. Springer, 2010.

[2] P. Goupil and A. Marcos, "The European ADDSAFE project: Industrial and academic efforts towards advanced fault diagnosis," Control Engineering Practice, vol. 31, pp. 109-125, 2014.

[3] P. Goupil, J. Boada-Bauxell, A. Marcos, P. Rosa, M. Kerr, and L. Dalbies, "An overview of the FP7 RECONFIGURE project: Industrial, scientific and technological objectives,' in SAFEPROCESS '15, 2015.

[4] T. Tucker, Touchdown: the development of propulsion controlled aircraft at NASA Dryden. Monographs in Aerospace History, 1999.

[5] F. Burcham, J. Burken, and T. A. Maine, "Flight testing a propulsion-controlled aircraft emergency flight control system on a F-15 airplane," in AIAA Biennial Flight Test Conference, no. AIAA 94-2123-CP, 1994.

[6] K. Masui and Y. Tsukano, "Development of a new in-flight simulator MuPAL- $\alpha$," AIAA paper 2000-4574, Aug. 2000.

[7] M. Sato and A. Satoh, "Flight control experiment of multipurpose-aviation-laboratory- $\alpha$ in-flight simulator," Journal of Guidance, Control, and Dynamics, vol. 34, 2011.

[8] H. Alwi, C. Edwards, O. Stroosma, J. Mulder, and M. Hamayun, "Real-time implementation of an integral sliding mode fault tolerant control scheme for LPV plants," IEEE Trans Ind Elect, vol. 62(6), pp. 3896-3905, 2015.

[9] M. T. Hamayun, C. Edwards, and H. Alwi, "A fault tolerant control allocation scheme with output integral sliding modes," Automatica, vol. 49(6), pp. 1830-1867, 2013.

[10] H. Alwi and C. Edwards, "Development and application of sliding mode LPV fault reconstruction schemes for the ADDSAFE Benchmark," Control Engineering Practice, vol. 31, pp. 148-170, 2014.

[11] B. Vanek, A. Edelmayer, Z. Szabo, and J. Bokor, "Bridging the gap between theory and practice in LPV fault detection for flight control actuators," Control Engineering Practice, vol. 31, pp. 171-182, 2014.

[12] J. Mohammadpour and C. W. Scherer, Control of Linear Parameter Varying Systems with Applications. Springer, 2012.

[13] H. Alwi, C. Edwards, and C. P. Tan, Fault Detection and Fault-Tolerant Control Using Sliding Modes. Springer, 2011.

[14] M. T. Hamayun, C. Edwards, and H. Alwi, Fault tolerant control schemes using integral sliding modes. Springer, 2016.

[15] H. Alwi and C. Edwards, "Fault tolerant control using sliding modes with on-line control allocation," Automatica, vol.44, pp.1859-66, 2008.

[16] O. Harkegard and S. Glad, "Resolving actuator redundancy - optimal vs. control allocation,” Automatica, no. 41, pp. 137-144, 2005.

[17] C. Edwards and S. K. Spurgeon, Sliding Mode Control: Theory and Applications. London, U.K.: Taylor \& Francis, 1998.

[18] D. Rotondo, F. Nejjari, V. Puig, and J. Blesa, "Model reference FTC for LPV systems using virtual actuator and set-membership fault estimation," Int. J. Robust Nonlinear Control, vol.25, pp.753-60, 2015.

[19] A. Levant, "Robust exact differentiation via sliding mode technique," Automatica, vol. 34, pp. 379-384, 1998.

[20] M. Sato and A. Satoh, "Simultaneous realization of handling and gust responses: In-flight simulator controller design," Journal of Guidance, Control and Dynamics, vol. 31, 2008.

[21] M. Sato, "Robust model-following controller design for LTI systems affected by parametric uncertainties: a design example for aircraft motion," Int. Journal of Control, pp.689-704, vol. 82(4), 2009.

[22] V. Utkin, Sliding modes in control and optimization. Springer, 1992. 